\title{
Dynamic optimization of the transmission efficiency between the solid state microwave sources and the microwave applicator
}

\author{
Zuber, S. ${ }^{\mathbf{a}^{*}}$; Joss, M. ${ }^{\mathbf{a}}$; Tresch, S. ${ }^{\mathbf{b}}$; Kleingries, M. ${ }^{\mathbf{b}}$ \\ ${ }^{a}$ Institute for electrical engineering. Lucerne University of Applied Sciences and Arts, Lucerne, \\ Switzerland \\ ${ }^{\mathrm{b}}$ Institute for mechanical engineering and energy technology. Lucerne University of Applied Sciences \\ and Arts, Lucerne, Switzerland
}

*E-mail of the corresponding author: simon.zuber.01@hslu.ch

\begin{abstract}
Microwaves are a fast way to dry moist goods through volumetric heating. During the drying process, materials change their electrical properties. As a result, the impedances at the feed port of the applicator will change and the microwave source is not matched anymore. The amount of reflected power increases and the process efficiency reduces. New semiconductor high power sources can perform a dynamic impedance matching. A lab scaled functional model with two sources was designed and realized. For measuring the scattering parameters during the process run, an embedded two-port vector network analyzer was added. Measurement results confirm the feasibility of the concept.
\end{abstract}

Keywords: Microwave drying, dynamic efficiency optimization, multichannel feed, solid state based microwave source 


\section{Introduction}

Microwaves (mw) allows a volumetric heating method used to dry moist goods. The volumetric heating is faster than conventional drying as soon as the surface of the good becomes dry and the core is still wet [1]. The most common method for an mw source in industrial applications today is the magnetron. It builds on proven technology, is costeffective and can also produce power in the $\mathrm{kW}$ range. But it also has some disadvantages such as the need of a high tension power supply and an output signal at a fix frequency. For optimum power transfer, the source is impedance matched to the load. However, during the drying process, materials change their electrical properties. And this will change the wave distribution inside the microwave applicator too. As a result, the impedance at the feed port of the applicator will change and the source is not matched anymore. The amount of reflected power increases and the process efficiency reduces. Figure 1 shows the measured reflected power in percentage in relation to the moisture content in a drying process. Underneath a certain moisture content, the reflected power increases. This is led by means of a circulator to an artificial load and converted into heat.

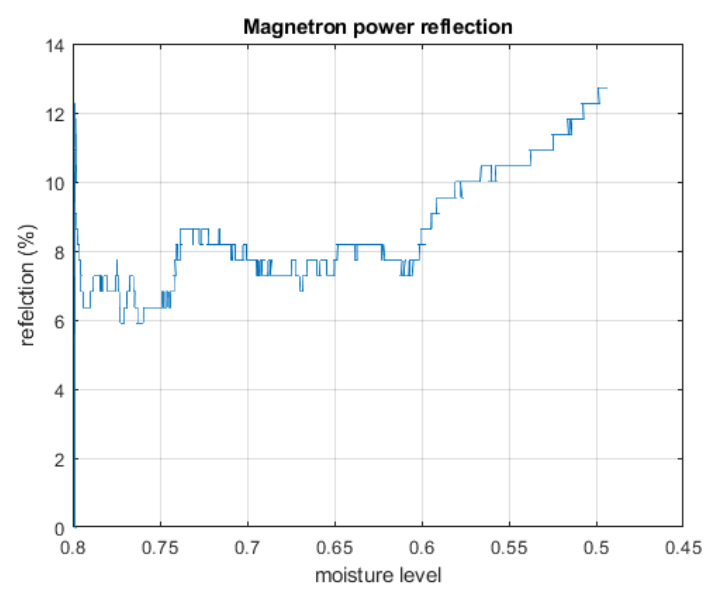

Fig. 1 increasing power reflection of a magnetron heating system due to load change

Frequency synthesizers and semiconductor power amplifiers for the $2.45 \mathrm{GHz}$ ISM band are becoming available for industrial applications. An mw source based on these components is able to change the frequency and phase of the created signal. This enables dynamic impedance matching, based on a search for the best frequency and phase condition.

Three dimensional finite element electromagnetic field simulations of several microwave chambers and drying goods have shown, that two feed ports are especially a benefit in a drying process [2]. This comes from the fact that a two port applicator allows a smoother electromagnetic field distribution, even if the volume of the good is shrinking over the drying process. In addition, during the drying process the impedance matching of two ports must be adapted continuously. Simulation results presented in previous papers showed that the optimum frequency and the optimal phase shift between two feeds can be estimated by means 
of complex scattering parameters [3] [4]. The objectives of our work were to verify the various simulation results by means of a lab scaled functional model of a two port applicator. In addition, we show the feasibility of a tunable mw source based on semiconductor components. Such a source is a prerequisite for searching the optimum operating point in the drying process.

Chapter two lists the applied methods and materials we used in our work. Chapter three describes the measurement and optimization algorithm. In the following chapter four, the system architecture of the two port mw source is explained. In chapter five measurement results are explained and discussed. The final chapter six contains the conclusion and hints for further work.

\section{Materials and Methods}

For the functional model of a semiconductor based two port mw source with frequency synthesizer, phase adaptation and embedded vector network functionality for measuring the scattering parameters (s-parameter), a suitable printed circuit board based microwave circuit was designed and produced. Although the available microwave output power was limited to $250 \mathrm{~mW}$ the measurement principles and the verification of the optimization algorithm could be done.

The two port mw source was connected to the applicator by means of WR340 waveguides. The applicator is made of aluminum and has a box type shape with the size of $0.61 * 0.69 * 0.51$ m. It works as a multimode oven [1]. The test good is placed on a weighing system in the center of the applicator, which allows to continuously weight the good during the drying process. In order to avoid the condensation of water vapor on the walls of the applicator, a ventilation system was installed.

In order to check the quality of the measured s-parameters with the built-in measuring device, all parameters were additionally measured with a Rhode \& Schwarz commercial network analyzer.

The used test good was a mixture of flour and water.

\section{Algorithm for efficiency calculation}

The overall efficiency $\eta_{\text {tot }}$ of a microwave heating system depends on various efficiency factors of the subsystems.

$$
\eta_{\text {tot }}=\eta_{\text {gen }} \eta_{\text {feed }} \eta_{\text {trans }} \eta_{\text {appl }}
$$

The efficiency of the microwave source $\eta_{\text {gen }}$ is mainly determined by the efficiency of the solid state power amplifier. The factor of feeder network efficiency $\eta_{\text {feed }}$ covers the losses of coupling elements, waveguides, power combiner and feeder antennas. The efficiency 
factor of transmission $\eta_{\text {trans }}$ is a figure of how good the impedance matching works. The efficiency factor $\eta_{\text {appl }}$ from the applicator describes the losses due to heating up the metal walls by means of electrical induction.

The focus of our work is the optimization of $\eta_{\text {trans }}$. This factor strongly dependents on the impedance matching between the feeder and the applicator with its drying good. For optimizing the transmission efficiency, we define two ways how to measure and calculate it. Both methods are applicable for a microwave applicator with two feeds.

The first method is suitable if both sources are simultaneously active and the electromagnetic fields are overlapped. The efficiency is calculated from the ratios between the reflected waves $b_{1,2}$ and the input waves $a_{1,2}$ at the individual feeder ports of the microwave applicator. The indices gives the individual port number. Because these waves represent voltage waves, the square of them are proportional to the power which leads directly to the needed power efficiency figure. The wave $b_{1}$ is a superposition of the reflected wave at port one and the through connection part from port two. In the same way the wave $b_{2}$ consists of the reflected wave at port two and the part which is transferred from port one. $\eta_{\text {trans }}$ will be maximized if the individual wave reflection at the ports can be minimized and the feed trough parts can be minimized. To find the optimal operation point the measurement and calculation for $\eta_{\text {trans }}$ must be done separately for every frequency and phase difference between the two sources.

$$
\eta_{\text {trans }}=1-\frac{1}{2}\left|\frac{b_{1}}{a_{1}}\right|^{2}-\frac{1}{2}\left|\frac{b_{2}}{a_{2}}\right|^{2}
$$

The second method is based on the s-parameters and thus only on source must be active during the measurement process. This method is more complex but needs less measurements [4]. This is possible because the phase information for the superposition of the two sources is included in the s-parameter. The s-parameter is defined as a ratio between an input wave $a$ and a reflected wave $b$. The index gives the individual port number. The first index of an s-parameters gives the number of the destination port and the second index the number of the source port. The s-parameters are complex numbers.

$$
\begin{aligned}
& s_{11}=\frac{b_{1}}{a_{1}}, \text { with } a_{2}=0 \\
& s_{12}=\frac{b_{1}}{a_{2}}, \text { with } a_{1}=0 \\
& s_{21}=\frac{b_{2}}{a_{1}}, \text { with } a_{2}=0 \\
& s_{22}=\frac{b_{2}}{a_{2}}, \text { with } a_{1}=0
\end{aligned}
$$


The transmission efficiency for a desired phase difference $\Delta \varphi$ can now be calculated by

$$
\eta_{\text {trans }}=1-\frac{1}{2}\left|s_{11}+s_{12} e^{j \Delta \varphi}\right|^{2}-\frac{1}{2}\left|s_{22}+s_{21} e^{-j \Delta \varphi}\right|^{2}
$$

For finding the optimal operating point with the highest $\eta_{\text {trans }}$, measurements must be done over all frequencies and calculations over all phases and frequencies.

\section{Architecture of the system}

To proof the concept that a microwave heating system based on semiconductor components allows a high transmission efficiency for several goods and moisture levels, a test hardware was built. The architecture of the system is shown in figure 2 .

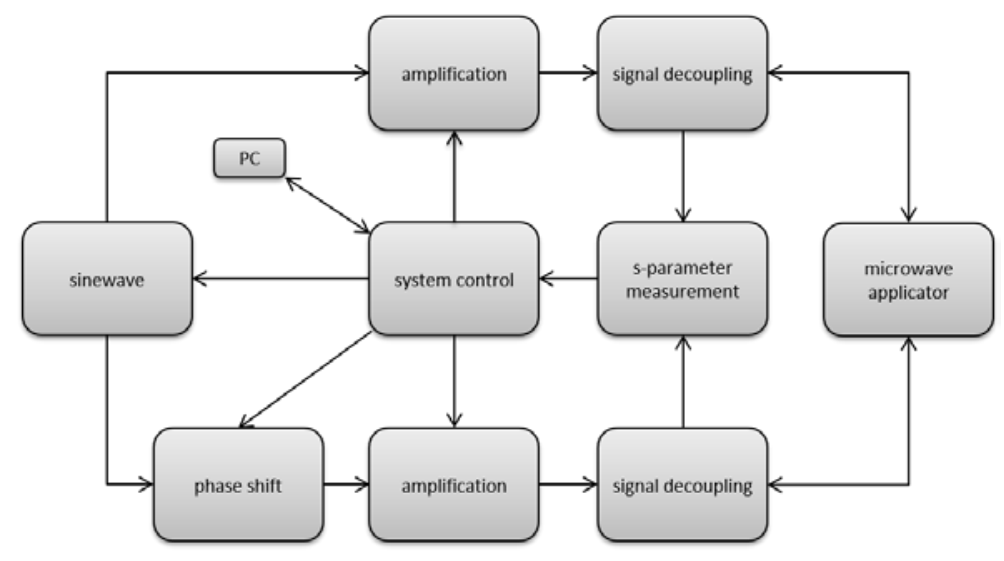

Fig. 2 block diagram of the functional prototype

The two sinewave signals are created with high performance, wideband rf synthesizers LMX2582 from Texas Instruments. This synthesizers are able to adjust both the output frequency and phase with a 32-bit resolution.

As for proofing the concept a high power is not needed, the two sinewave signals are only amplified with a driver amplifier MGA-30489 from Avago. The amplifier delivers $250 \mathrm{~mW}$ of microwave power.

For executing the s-parameter measurement the forward and the reflected signal wave must be separated first. A high power integrated directional coupler X3C26P1 from Xinger is used for this task. Only $-30 \mathrm{~dB}$ of the individual signal waves are decoupled for the measurement.

The s-parameter measurement is done with an rf gain and phase detector AD8302 from Analog Devices. In the system control part a microcontroller is used for calculating the efficiency parameter. It also calibrates the measured values [5] and controls the other subsystems such as sinewave generation, phase shifting and amplification. 


\section{Results and Discussion}

To validate the concept and the prototype hardware several measurements with various material mixtures as indicated in table 1 have been done.

Table 1. Material mixtures

\begin{tabular}{lll}
\hline Moisture level: & Water: & Flour: \\
\hline $80 \%$ water & $400 \mathrm{~g}$ & $100 \mathrm{~g}$ \\
$50 \%$ water & $100 \mathrm{~g}$ & $100 \mathrm{~g}$ \\
$20 \%$ water & $25 \mathrm{~g}$ & $100 \mathrm{~g}$ \\
\hline
\end{tabular}

For the different measurement campaigns the frequency range from $2.4 \mathrm{GHz}$ till $2.5 \mathrm{GHz}$ with a step size of $1 \mathrm{MHz}$ was swept through. For each frequency point the s-parameters where measured. With these set of s-parameters the efficiency for all frequencies and phase differences with a resolution of one degree where calculated with (7). Subsequently the highest efficiency $\eta_{\text {trans }}$ over all phase differences is searched for each frequency. This maximum efficiency for all frequencies of the three moisture levels are depicted in figure 3.

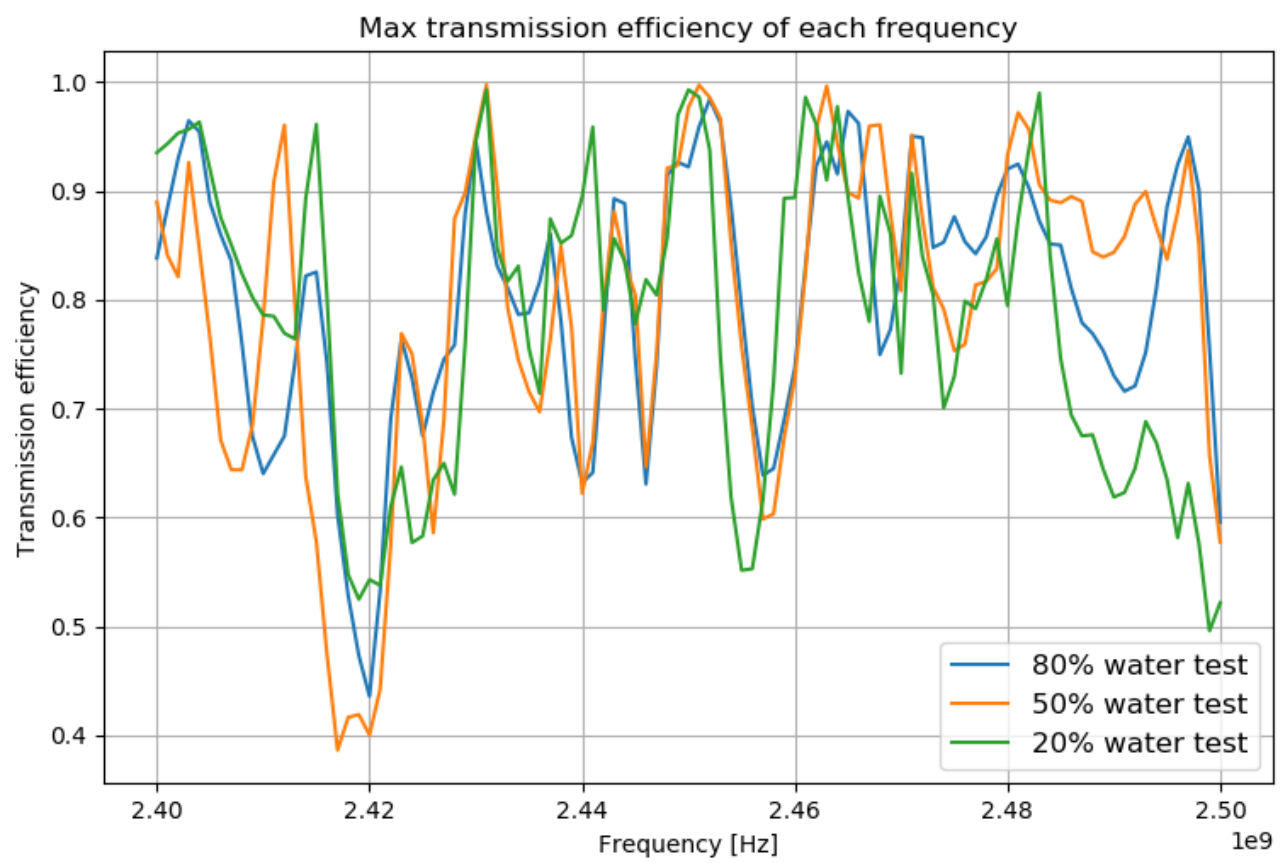

Fig. 3 calculated transmission efficiencies based on s-parameter measurements 
The optimal frequency and phase difference changes during the drying process. Table 2 shows the best values found for the three moisture levels. The efficiency value remains almost on the same high level over all three measurement series.

Table 2. Ideal parameters

\begin{tabular}{llll}
\hline moisture level: & best efficiency: & frequency: & phase difference: \\
\hline $80 \%$ water & 0.984 & $2.452 \mathrm{GHz}$ & $190^{\circ}$ \\
$50 \%$ water & 0.997 & $2.451 \mathrm{GHz}$ & $202^{\circ}$ \\
$20 \%$ water & 0.993 & $2.450 \mathrm{GHz}$ & $201^{\circ}$ \\
\hline
\end{tabular}

For validating the measurement accuracy of the prototype hardware, a comparison of the calculated efficiency values have been done. The results are illustrated in figure 4 . The blue color curve shows the calculated values with (7) based on s-parameter measurements done with the vector network analyzer ZVL6 from Rhode\&Schwarz. The yellow curve shows the results calculated with (7) based on s-parameter measurements of the prototype hardware. And the green curve are the results based on the calculation method of (2). All three methods are very close to each other if the reflected power is small. However, the calculated efficiency values differ with increasing reflections.

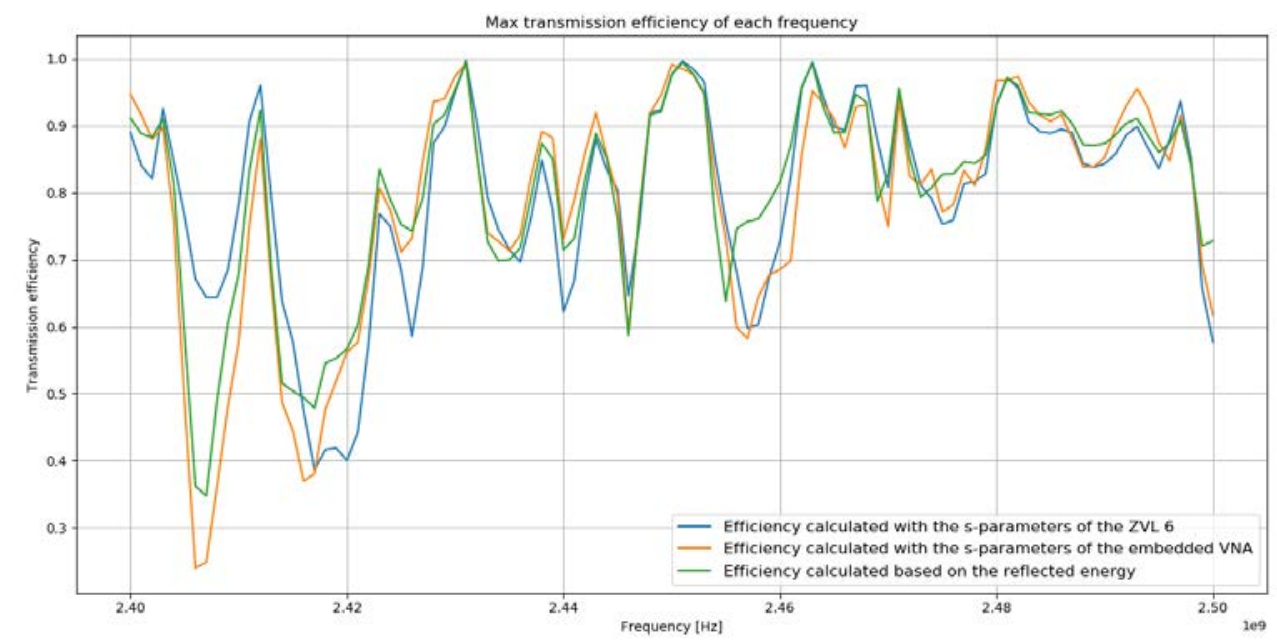

Fig. 4 comparison of measurement methods (mixture with $50 \%$ moisture level) 


\section{Conclusions}

A microwave heating system is a fast and efficient way to dry goods. The classical solution based on a magnetron as microwave source gets problems to put the energy into the dry good if electrical properties changes at low moisture levels. A heating system based on a semiconductor solution as microwave source shows promising results. Such a system allows the adaptation of operation frequency and phase and offers an impedance tuning function during the running process. With an s-parameter measurement the optimal frequency point and phase difference can be determined and allows a maximization of the transmission efficiency. For a proof of concept, a lab scaled functional model was developed. An embedded system with two frequency synthesizers generates the two feeder signals. The power, frequency and phase of the signals can be controlled by means of a microcontroller system. For measuring the s-parameters during the process, an embedded two-port vector network analyzer was added. The obtained measurement results fulfill the expectations.

\section{References}

[1] R. Meredith, Engineers' Handbook of Industrial Microwave Heating, The Institution of Engineering and Technology, 2007, pp. 54-63.

[2] S. Zuber and M. Joss, "Multichannel Microwave Applicator," Lucerne, Switzerland, 2017.

[3] P. Korpas, A. Więckowski and M. Krysicki, "Effects of applying a frequency and phaseshift efficiency optimisation algorithm to a solid-state microwave oven," Institute of Radioelectronics, Warsaw University of Technology, 2016.

[4] A. Wieckowskia, P. Korpas, M. Krysickia, F. Dughiero, M. Bullo, F. Bressan and C. Fager, "Efficiency optimization for phase controlled multi-source microwave oven," Institute of Radioelectronics, Warsaw University of Technology, Warsaw, Poland, 2014.

[5] T. Valeria, A. Ferrero and M. Sayed, Modern RF and Microwave Measurement Techniques, Cambridge University Press, 2013. 